\title{
Intensive care of the patient with acute myocardial infarction
}

\author{
M. W. MCNicOL \\ Central Middlesex Hospital, London, N.W.1
}

ACUTE myocardial infarction has an unknown total mortality; of the patients admitted to hospital $20-40 \%$ die. By the intensive care of these patients in special units it has been possible to reduce that mortality to $15 \%$ (Day, 1965). Even without special units significant reduction in mortality can be obtained. We have been able to reduce mortality on our own wards which have no coronary care unit from $34 \%$ to $22 \%$, but this is an inefficient process and the improvement is gained at the cost of much staff time and effort. Reduction of the high mortality of myocardial infarction depends on the seeking out of known dangerous complications and their energetic treatment, both of which are made much easier in a coronary care unit. The period during which very intensive therapy is required is short. Half of the hospital deaths occur within $72 \mathrm{hr}$, and present practice is to restrict intensive care to that time. The apparent irreducible mortality with existing methods of treatment is $15 \%$ and the object of treatment should be to reduce mortality to that level. In this paper I will discuss the complications of myocardial infarction which increase mortality, and the means of detecting and treating them.

\section{Causes of increased mortality}

The complications of myocardial infarction associated with an increase in mortality are: arrhythmias, hypotension, peripheral circulatory failure, and cardiac failure. These complications may occur together, particularly in the more severely ill patients. Their correction does improve prognosis, and they should be regarded as important correctible abnormalities, not merely as symptoms of serious illness. Successful treatment is based on early recognition and vigorous management. Clinical awareness and thorough treatment are required. Complex methods and equipment are very helpful, but not essential.

\section{Effect of arrhythmias on mortality}

The incidence of arrhythmia in acute myocardial infarction has only been accurately assessed since methods for continuous electrocardiographic monitoring have become available. Julian, Valentine \& Miller (1964) found 240 arrhythmias in 100 consecutive patients; 138 of these $(57 \%)$ were serious arrhythmias associated with an increased mortality. Most of the arrhythmias were detected during the first $48 \mathrm{hr}$ after admission to hospital (see Table 1). One-third of all arrhythmias and one-third of the serious arrhythmias were detected before monitoring had started, and most of the remainder during the subsequent 48 -hr period of monitoring. Of the

TABLE 1

Time of incidence of arrhythmia in acute myocardial infarction in 100 patients (data of Julian et al., 1964)

\begin{tabular}{lcccc}
\hline & $\begin{array}{c}\text { Before } \\
\text { monitoring }\end{array}$ & $\begin{array}{c}\text { During } \\
\text { monitoring }\end{array}$ & $\begin{array}{c}\text { After } \\
\text { monitoring }\end{array}$ & Total \\
\hline $\begin{array}{l}\text { All } \\
\text { arrhythmias }\end{array}$ & $\underbrace{30 \%}_{92 \%}$ & $82 \%$ & $100 \%$ \\
$\begin{array}{l}\text { Serious } \\
\text { arrhythmias }\end{array}$ & $\underbrace{34 \%}_{92 \%} 58 \%$ & $8 \%$ & $100 \%$ \\
& & & $=138$ \\
\hline
\end{tabular}

arrhythmias detected $92 \%$ occurred during the first 2 days, and only $8 \%$ during the rest of the period in hospital. The decrease in incidence of the arrhythmias after $48 \mathrm{hr}$ is partly an artefact due to less intensive observation, and more prolonged monitoring will show a higher incidence of arrhythmia after $48 \mathrm{hr}$. Some of these late occurring arrhythmias may be important; ventricular fibrillation may occur 10-14 days after infarction in patients whose prognosis otherwise appears good. Different methods of monitoring will be required to detect these episodes. Until these are available it seems reasonable to accept $48-72 \mathrm{hr}$ as the period in which most serious arrhythmias occur. Particular care during this period is essential, and continuous monitoring is desirable. When monitoring is not possible, close observation during this time is a partial substitute.

The serious arrhythmias and the mortality associated with them are listed in Table 2 (derived from Julian's data). Two common arrhythmias are associated with a definite increase in mortalitysinus tachycardia and multiple ventricular ectopics. Sinus tachycardia (rate above $100 / \mathrm{min}$ ) occurred in forty-three patients and was associated with a $41 \%$ 
mortality. Other less common arrhythmias were associated with higher mortality, notably ventricular fibrillation and ventricular tachycardia with a mortality above $66 \%$. Complete heart block in this series had a relatively low mortality $-37 \%$; the mean mortality of reported cases is $54 \%$ (Epstein et al., 1966). Paroxysmal atrial tachycardia also had a relatively high mortality $(50 \%)$.

\section{TABLE 2}

Incidence of serious arrhythmia in acute myocardial infarction in 100 patients (data in Julian et al., 1964)

\begin{tabular}{lrr}
\hline \multicolumn{1}{c}{ Arrhythmia } & No. & Mortality \\
\hline Sinus tachycardia (100/min) & 43 & $19(44 \%)$ \\
Frequent ventricular ectopics (1 in 10) & 32 & $13(41 \%)$ \\
Ventricular fibrillation & 10 & $9(90 \%)$ \\
Complete heart block & 8 & $3(37 \%)$ \\
Ventricular tachycardia & 6 & $4(67 \%)$ \\
Paroxysmal atrial tachycardia & 4 & $2(50 \%)$ \\
All patients & 100 & 31 \\
\hline
\end{tabular}

Sinus tachycardia and frequent ventricular ectopics were detected before monitoring in $52 \%$ of cases, but of the more dangerous arrhythmias only $28 \%$ were detected before monitoring. For some of these, notably ventricular fibrillation and ventricular tachycardia, the detection rate will remain low unless continuous monitoring is used. Quick detection of these arrhythmias is one of the first essentials of successful intensive therapy.

\section{Effect of functional complications on mortality}

The functional complications of myocardial infarction-hypotension, peripheral circulatory failure and cardiac failure-also have a marked effect on prognosis (McKenzie et al., 1964; McNicol et al., 1965). The association of hypotension and peripheral circulatory failure characterizes 'shock', which is usually associated also with cardiac failure. In myocardial infarction the state of the peripheral circulation is a more reliable index of shock than the blood pressure. A few patients with hypotension have a normal or increased cardiac output with a decreased peripheral resistance. They usually have no cardiac failure and their prognosis is relatively good. The occurrence of hypotension then gives a misleading impression of serious illness. The blood pressure may also be misleading in the opposite direction - a previously hypertensive patient can have an apparently normal blood pressure despite peripheral circulatory failure and cardiac failure, and despite the 'normal' blood pressure have a poor prognosis. In our experience shocked patients with a bad prognosis have both cardiac failure and peripheral circulatory failure, and the level of the blood pressure is not particularly helpful. This is illustrated by the mortality in a personally observed series of $\frac{\mathbb{}}{2}$

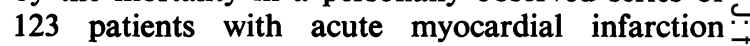
(Table 3). Mortality in patients with both hypo- $\frac{\overrightarrow{\bar{s}}}{+}$ tension and cardiac failure was higher than those $-\overrightarrow{0}$ with hypotension alone.

TABLE 3

Clinical grouping and mortality in acute myocardial infarction

\begin{tabular}{|c|c|c|}
\hline Group & No. & Mortality (\%) \\
\hline $\begin{array}{l}\text { Uncomplicated } \\
\text { Hypotension } \\
\text { Hypotension and cardiac failure } \\
\text { Cardiac failure }\end{array}$ & $\begin{array}{r}28 \\
7 \\
26 \\
62\end{array}$ & $\begin{array}{r}0 \\
57 \\
85 \\
34\end{array}$ \\
\hline All patients & 123 & 38 \\
\hline
\end{tabular}

These patients were classified into four groups; $i$ uncomplicated myocardial infarction: myocardial $\mathcal{}$ infarction with hypotension: myocardial infarction 을 with hypotension and cardiac failure: myocardial infarction with cardiac failure, but without hypo- $\rightarrow$ tension. Cardiac failure was diagnosed if there were persistent basal crepitations or more obvious signs. $\vec{\bullet}$ It was present in $72 \%$ of the patients and is probably the commonest complication of myocardial infarction. It is a significant complication. Completely uncomplicated myocardial infarction had no mortality, but in the presence of cardiac failure mortality rose to $34 \%$. Cardiac failure is a very important complication of myocardial infarction-important both because it is very frequent and because it can be treated.

Cardiac failure and fall in cardiac output both impair oxygen uptake in the lung and cause arterial hypoxaemia. The exact mechanism has not yet been 흘 fully elucidated, but the evidence suggests that disturbance of ventilation-perfusion relationships is the $\frac{5}{3}$ main factor (McNicol et al., 1965). This is amenable to treatment by increasing inspired oxygen concentration. Diuretics also improve oxygen uptake by the lung when heart failure is present. The correlation between the severity of hypoxaemia and the clinical $D$ signs of cardiac failure is poor. This was commented on as long ago as 1954 in a paper entitled 'The lack $N$ of correlation between rales and arterial oxygen $N$ saturation in patients with pulmonary congestion 0 and oedema' (Vitale, Dumke \& Comroe, 1954), but $\stackrel{\omega}{9}$ many people still fail to realize that the correlation is poor and underestimate the significance of $\stackrel{\varrho}{\complement}$ apparently minor signs in the chest. In our patients there was no parallel between clinical grading and arterial blood gas tensions, and a profound fall in arterial oxygen tension was as often associated with trivial signs as with florid pulmonary oedema. Hypoxaemia is related to mortality (Table 4); when arterial oxygen tension is below $50 \mathrm{mmHg} 77 \%$ die. Although some clinical sign is usually present, 8 
measurement of arterial oxygen tension will reveal some patients to be at high risk who would otherwise not have been suspected.

\section{TABLE 4}

Hypoxaemia, acidosis and mortality in myocardial infarction

\begin{tabular}{lcc}
\hline & No. & Mortality (\%) \\
\hline Oxygen tension $<50 \mathrm{mmHg}$ & 26 & 77 \\
$\mathrm{pH}<7 \cdot 30$ & 12 & 84 \\
Bicarbonate $<15 \mathrm{mEq} / \mathrm{l}$ & 16 & 75 \\
All patients & 123 & 38 \\
\hline
\end{tabular}

Acidosis is a symptom of tissue hypoxia and is found in patients with peripheral circulatory failure. In our patients it was present mainly in those with hypotension and cardiac failure, and its presence could usually be suspected on clinical grounds. Those with severe acidosis $(\mathrm{pH}$ less than 7.30) are at high risk-mortality $84 \%$. Exact assessment is important, for correction of acidosis has some influence on survival.

\section{Treatment}

Intensive observation must be accompanied by intensive treatment. Correctible arrhythmias must be corrected, and the function of the heart and the circulation must be supported.

The most serious complication of myocardial infarction is cardiac arrest, and though intensive therapy may reduce its incidence it is always liable to occur. Any intensive therapy unit for myocardial infarction must have good arrangements for the treatment of cardiac arrest. Our own arrangements have been described by Kirby (1967). We use routine methods of mouth-to-mouth breathing and external cardiac massage. We correct acidosis early and ventilate as soon as possible with pure oxygen. A synchronous d.c. defibrillator is used, and is available for treatment of other arrhythmias. For patients in heart block or asystole we use internal pacing with a bipolar catheter electrode. All members of medical and nursing staff are trained in the techniques of emergency resuscitation, but definitive management is limited to a small group.

Despite considerable effort our initial success rate in acute myocardial infarction was low- $-10 \%$ longterm survivors in fifty attempts. Recently our results have improved greatly. We admit to our own wards about half of the patients admitted to the hospital with acute myocardial infarction; the remainder are scattered through the other three medical units. During 1965 we paid particular attention to the patients on our own wards. Resuscitation was attempted on a total of thirty-seven patients with acute myocardial infarction; of these nineteen were on our own wards and eight survived. There were no survivors from the eighteen attempted resuscitations on other wards. The high long-term survival rate on our own wards $(42 \%)$ is due to earlier diagnosis and more effective therapy, and this is due to the concentration of patients, resources and interest. Such concentration is essential for the intensive therapy of myocardial infarction, and would be best provided in a coronary care unit.

Serious arrhythmias other than cardiac arrest. Sinus tachycardia reflects the severity of the infarction or its complications, and there is no specific treatment. Frequent ventricular ectopics predispose to ventricular tachycardia and ventricular fibrillation; any patient with these arrhythmias should be closely observed especially if $\mathbf{R}$ on $\mathbf{T}$ ectopics are present. Antiarrhythmic drugs should be given and either procaine amide or propranolol are the drugs of choice. Procaine amide should be used with very great care if there is a conduction defect. Propranolol should be used with great care in the presence of hypotension or cardiac failure; atropine should always be available to reverse its action if it is given intravenously, and if cardiac failure is marked digitalis should be given at the same time. No adequate comparative assessment between procaine amide and propranolol has been made and the choice between them is dictated by individual preference. We use oral propranolol in doses of up to $30 \mathrm{mg}$ every $4 \mathrm{hr}$.

Supraventricular arrhythmias should be treated only when the ventricular rate is rapid (over $100 / \mathrm{min}$ ). When there is paroxysmal atrial tachycardia the possibility of digitalis overdosage should always be considered; if this is present the treatment is with propranolol. If the arrhythmia is not due to digitalis, the patient should be digitalized. For persistent atrial tachycardia resistant to digitalis and propranolol, cardioversion should always be considered; in our experience it is effective and safe. Atrial fibrillation should be treated with digitalis only if it is persistent and the rate is rapid.

Ventricular tachycardia must be urgently treated with either intravenous procaine amide (up to $1 \mathrm{~g}$ ) or propranolol (up to $5 \mathrm{mg}$ ) with continuous control of ECG and blood pressure. If propranolol is used atropine must be always available. If reversion to sinus rhythm is not quickly obtained with these drugs, cardioversion must be used without delay.

Complete heart block presents a considerable therapeutic problem. The slow heart rate predisposes to shock and heart failure, and the incidence of ventricular fibrillation is high. Treatment with sympathomimetic drugs usually speeds the heart, but carries the risk of producing ventricular fibrillation. Sympathomimetics should be given if the heart rate remains below $60 / \mathrm{min}$, and if no other form of treatment is available. Pacing with an electrode 
catheter seems a more rational approach, but it requires more complex resources, and adequate information on results in sufficient numbers of patients is not yet available. Our own preliminary results are encouraging. Partial heart block does not require treatment.

Recurrent episodes of ventricular fibrillation can cause much trouble. They are often preceded by multiple ventricular ectopics. Full doses of intravenous propranolol or procaine amide should be given, but these drugs are not always effective. Pacing of these patients may then prove very helpful (Sowton, Leatham \& Carson, 1964). Tachycardias can also be suppressed by paired or triple pulse pacing (Bayley \& Lightwood, 1966), but the exact place of this treatment has not been established.

\section{Treatment of cardiac failure}

Cardiac failure is dangerous both because it produces impairment of oxygen uptake in the lung, and because it produces poor tissue perfusion. It occurs in $75 \%$ of patients with myocardial infarction, and normally has a greater effect on oxygen uptake than on tissue perfusion. The hypoxaemia is always partially correctible by oxygen, and often is completely correctible (McKenzie et al., 1964; McNicol et al., 1965). High concentrations of oxygen should be used to obtain the maximum effect, and all patients with any sign of cardiac failure should have continuous oxygen therapy at an inspired concentration of more than $50 \%$. We use the M.C. mask at an oxygen flow rate of $61 / \mathrm{min}$; this gives a reasonable compromise between comfort and efficiency. Treatment with diuretics also improves the function of the oedematous lung, and diuretics should be given routinely in effective doses to all patients with cardiac failure. If cardiac failure is severe and persistent, digoxin and potassium supplements should be given. If there are ventricular ectopics propranolol should be combined with digoxin; this combination is said to preserve the positive inotropic action of digitalis, but prevent the effect of cardiac muscle irritability.

Shock and hypotension. As this subject is to be discussed in detail by Dr Nixon, I will only summarize our current practice. If hypotension is associated with peripheral vasodilation, a normal cardiac output and good tissue blood flow we give no treatment. Shock with poor peripheral circulation, hypotension and cardiac failure we treat urgently. Cardiac failure is usually present and the central venous pressure is usually raised; if it is low we transfuse to restore a normal pressure. For cardiac failure we give diuretics and oxygen at high concentration (70\% and over). Acidosis is common in these patients and is corrected by intravenous infusion of bicarbonate $(1 \mathrm{mEq} / \mathrm{ml})$. This raises the blood pressure (Neaverson, 1966) but probably not as a specific action. Though acidosis can be corrected without much difficulty, improvement is rarely striking.

All arrhythmias must be treated wherever possible, in particular any marked tachycardia or bradycardia. Arrhythmia can cause shock and often perpetuates it, and the effect of correction is often dramatic. If shock persists after these measures, the patient should be digitalized.

Only if there is no improvement and the patient seems seriously shocked should pressor drugs be used. Drugs with a purely peripheral constrictor action (alpha sympathomimetic action) are not helpful. Metaraminol has a direct cardiac stimulant action as well as a peripheral constrictor effect. It is our drug of choice, and if there is no response to it, further pressors are not used. Thus far the mortality in this group of patients remains high despite all forms of treatment.

\section{Other aspects of treatment of acute myocardial infarction}

Analgesics and sedatives are respiratory depressants which are liable to aggravate hypoxaemia, and may have undesirable effects on the circulation (Thomas et al., 1965). They should be used only for the relief of pain, and then only in the smallest effective doses. Routine 'sedation' should not be used.

Anticoagulants have little influence on the acute stage of myocardial infarction. We use them routinely only for thrombo-embolic complications, and after cardiac arrest.

Propranolol has been given prophylactically to all patients with myocardial infarction, and in a preliminary trial Snow (1965) reported very good results. This drug which is a beta adrenergic blocker stabilizes the muscle cell membrane; it also has a weak quinidine-like action on conduction (MoralesAguilera \& Vaughan Williams, 1965) and it also has a negative inotropic effect. It would be expected to have two effects in acute myocardial infarction, one beneficial by reduction of arrhythmia and the other harmful by depressing cardiac muscle function. The very great reduction in mortality in Snow's trial from $35 \%$ to near the present minimum mortality at $16 \%$ was therefore surprising. The design of the $\stackrel{\sim}{\circ}$ trial is open to some criticism, and as yet no proper trial has been conducted.* We do not think that propranolol is indicated as a routine treatment, and $\omega$ reserve it for the indications already described. It is possible that by combining propranolol with digoxin $\stackrel{\varrho}{C}$ therapeutic efficiency may be increased and potentially dangerous side effects reduced. Digoxin should always be given with propranolol if the patient is in heart failure.

The 'polarizing solution' of Sodi Pallares has been

\footnotetext{
* Since preparation of this paper a controlled clinical trial has shown that propranolol does not reduce mortality after myocardial infarction (Balcon et al., 1966).
} 
subjected to more satisfactory trial with apparently very good results (Mitra, 1965). This combination of glucose, potassium and insulin drives potassium into the muscle cell and so stabilizes the cell membrane and reduces the tendency to arrhythmia. It also has a positive inotropic effect. Though further investigation is required, this treatment has no apparent adverse side-effects, and must have a strong claim for routine use. An extended trial is at present being planned by the Medical Research Council.

\section{Summary}

Intensive therapy of the patient with acute myocardial infarction depends on a full assessment of cardiac rhythm and of the functional state of the heart and peripheral circulation. Assessment of arterial oxygenation and acid-base status are important. Any abnormalities discovered must be corrected. A good cardiac arrest service and some facilities for monitoring are essential. Centralization of treatment facilities and concentration of patients is most helpful. Restraint is necessary in the use of analgesics, sedatives and vasoconstrictors which have dangerous side-effects.

\section{Acknowledgments}

The author is supported by a grant from the Medical Research Council. He is indebted to the many colleagues who have helped to make the observations described in this paper, in particular to Dr B. J. Kirby.

\section{References}

Balcon, R., JewitT, D.E., Davies, J.P.H. \& ORAM, S. (1966) A controlled trial of propranol in acute myocardial infarction. Lancet, ii, 917.
Bayley, T.J. \& Lightwood, R. (1966) Double and triple pulse pacemaking in treatment of ventricular tachycardia. Lancet, i, 235.

DAY, H.W. (1965) Effectiveness of an intensive coronary care case. Amer. J. Cardiol. 15, 51.

Epstein, E.L., Coulshed, N., McKendrick, C.S., Clarke, J. \& Keams, W.E. (1966) Artificial pacing by electrode catheter for heart block or asystole complicating acute myocardial infarction. Brit. Heart J. 28, 546.

Julian, D.G., Valentine, P.A. \& Miller, G.G. (1964) Disturbances of rate rhythm and conduction in acute myocardial infarction: a prospective study of 100 consecutive unselected patients with the aid of electrocardiographic monitoring. Amer. J. Med. 37, 915.

KIRBY, B.J. (1967) Proc. roy. Soc. Med. (In press).

MCKenZIE, G.J., TAYLOR, S.H., Flenley, D.C., MCDONAld A.H., Staunton, H.P. \& Donald, K.W. (1964) Circulatory and respiratory studies in myocardial infarction and cardiogenic shock. Lancet, ii, 825 .

MCNicol, M.W., KIRBY, B.J., BHOOLA, K.D., Everest, M.E., Price, H.V. \& Freedman, S.F. (1965) Pulmonary function in acute myocardial infarction. Brit. med. J. ii, 1270.

MitrA, B. (1965) Potassium, glucose and insulin in treatment of myocardial infarction. Lancet, ii, 607.

Morales-Aguilera, A. \& Vaughan Williams, E.M. (1965) The effects on cardiac muscle of beta-receptor antagonists in relation to their activity as local anaesthetics. Brit. J. Pharmacol. 24, 332.

NeAVERSON, M.A. (1966) Metabolic acidosis in acute myocardial infarction. Brit. med. J. ii, 383.

SNOw, P.J.D. (1965) Effect of propranolol in myocardial infarction. Lancet, ii, 551.

Sowton, E., Leatham, A. \& Carson, P. (1964) The suppression of arrhythmias by artificial pacemaking. Lancet, ii, 1098.

Thomas, M., Malmcrona, R., Fillmore, S. \& ShillingFORD, J. (1965) Haemodynamic effects of morphine in patients with acute myocardial infarction. Brit. Heart J. 27, 863.

Vitale, A., Dumke, P.R. \& Comroe, J.H. (1954) The lack of correlation between rales and arterial oxygen saturation in patients with pulmonary congestion and oedema. Circulation, 10, 81 . 